Hydroécol. Appl. (1992) Tome 4 Vol 2, pp. 91-103

\title{
Le peuplement macrobenthique du port d'Alger: impact de la pollution
}

\section{Rebzani-Zahaf}

Université des Sciences et Technologies Houari Boumédiène (USTHB) Institut des Sciences de la Nature B.P. No 39 El Alia Alger

Résumé. - L'impact de la pollution sur le peuplement macrobenthique a été étudié durant un cycle saisonnier à travers l'analyse de 34 stations réparties dans les différents bassins du port (bassins Mustapha, Agha et Vieux Port).

Pour chacune de ces stations la composition faunistique, et la signification écologique de chaque espèce ou groupe d'espèce, ont permis de définir sept zones de pollution qui ont été délimitées dans les différents bassins.

Abstract. - Within the frame of a study on the benthic population of Algier port, 34 stations are analysed following a season cycle in the bassin of Mustapha, Agha and Vieux Port.

Southern portion of this port is more polluted than the normal one; the macrobenthic communities reflect this situation and 7 zones of decrease pollution are delimited in the different regions of Algier port.

\section{INTRODUCTION}

L'altération de l'environnement naturel notamment le milieu marin est progressivement devenue une préoccupation mondiale. Les rejets urbains et industriels deviennent de plus en plus importants, polluant la nature et constituant un danger croissant pour le milieu marin. Devant le développement industriel, la progression démographique mondiale et la grande densité des zones urbaines, l'hornme et ses activités contribuent énormément à l'installation et au développement de la pollution en mer.

Les mers et les océans, longtemps considérés comme un milieu purificateur ayant une capacité illimitée à "absorber" tous les rejets côtiers se sont avérés inefficaces devant l'importance des déchets déversés par l'homme et qui sont pour la plupart non biodégradables. Les milieux por- 
tuaires constituent des foyers importants de cette pollution.

Le port d'Alger, où convergent les rejets urbains d'une ville de près de deux millions d'habitants, est le siège d'activités industrielles, commerciales, de pêche et de plaisance.

L'étude de l'impact de ces rejets sur le milieu portuaire a été effectuée à travers l'analyse du peuplement macrobenthique.

Les espèces benthiques et plus particulièrement certains polychètes sont des indicateurs des conditions du milieu dans les zones plus ou moins polluées; ces espèces reflètent non seulement les conditions du milieu au moment de leur prélèvement, mais aussi celles qui existaient depuis le moment où elles se sont installées dans leur biotope; elle peuvent aussi cumuler et mémoriser des apports plus anciens (Hily, 1984).

\section{MILIEU ET MÉTHODES D'ÉTUDE} (Figures 1 et 2)

\section{Milieu}

Situé au Nord-Ouest de la baie d'Alger, le port qui représente une superficie de 184 ha est constitué de 3 bassins:

- Le bassin de Mustapha, d'une superficie de 75 ha, est un bassin à intense activité industrielle et commerciale, sa profondeur varie entre 7 et $11 \mathrm{~m}$.
- Le bassin de l'Agha est situé entre le bassin de Mustapha et celui du vieux port; sa profondeur est comprise entre 6 et $15 \mathrm{~m}$. C'est un bassin de transport de marchandises diverses qui offre un plan d'eau de 35 ha.

- Le bassin du Vieux Port, a une superficie de 74 ha et une profondeur de 7 à $20 \mathrm{~m}$; c'est un bassin à fonctions multiples : transport de marchandises et de voyageurs, plaisance, pêche et radoub.

Afin de couvrir l'ensemble du port, 34 stations ont été positionnées au fond des darses, à la sortie des darses et dans la zone d'évolution des navires de chaque bassin (Mustapha: 13 stations; Agha: 7 stations; Vieux Port: 14 stations).

\section{Méthodes d'étude}

Les prélèvements ont été réalisés trimestriellement, suivant un cycle saisonnier depuis décembre 1983 à avril 1985.

Les prélèvements du peuplement macrobenthique ont été effectués à l'aide d'une benne Orange Peel $\left(1 / 12 \mathrm{~m}^{2}\right)$; trois coups de benne ont été réalisés à chaque station et à chaque saison. Le sédiment recueilli a été tamisé sur maille de $1 \mathrm{~mm}$; le refus de tamis renfermant la macrofaune est fixé au formol à bord puis trié et déterminé au laboratoire.

Le peuplement a été étudié selon la méthode mise au point par Picard (1965) qui caractérise le peuplement par sa richesse spécifique et le nom- 


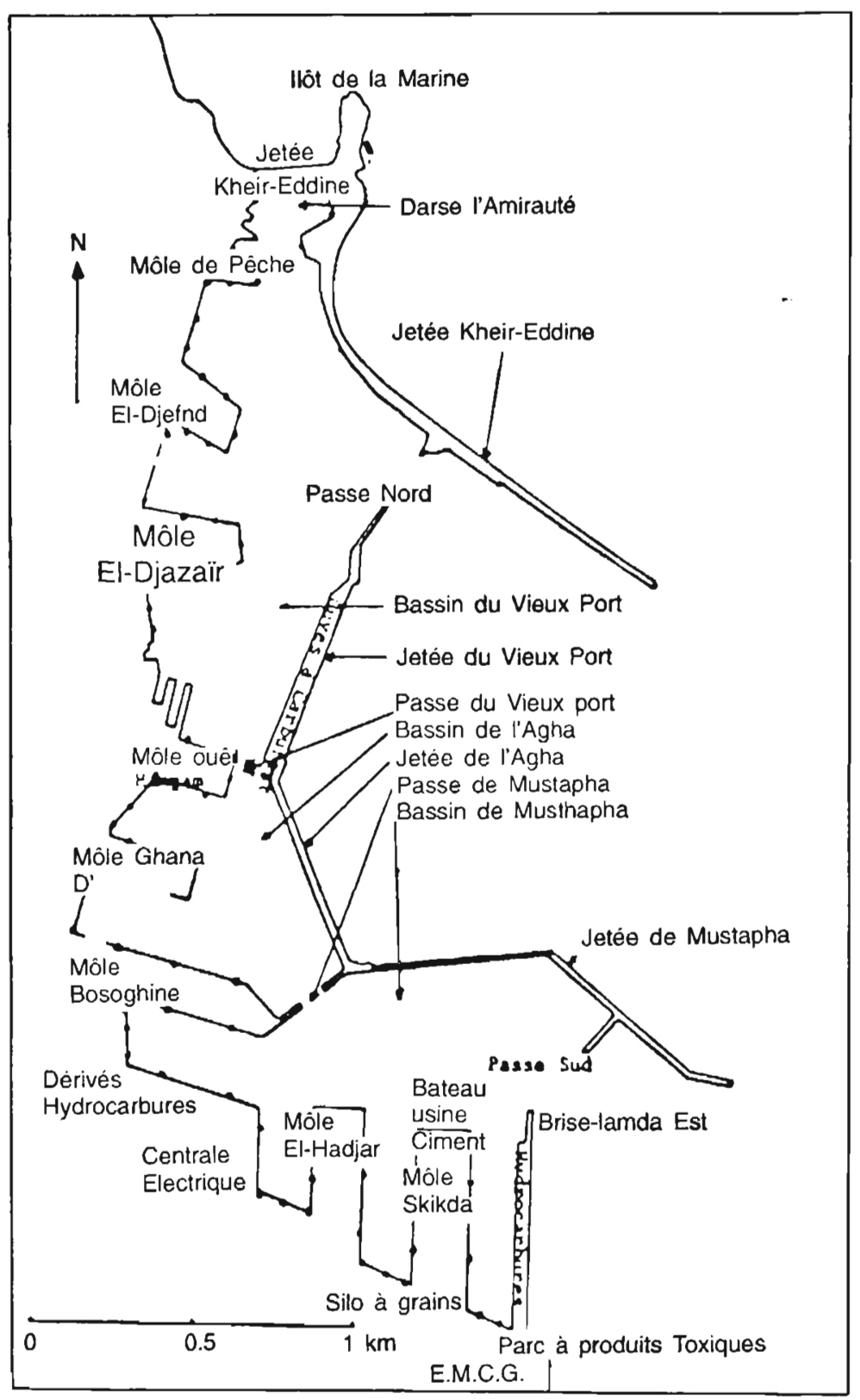

Fig. 1. - Présentation du port d'Alger ( $:$ égouts). 


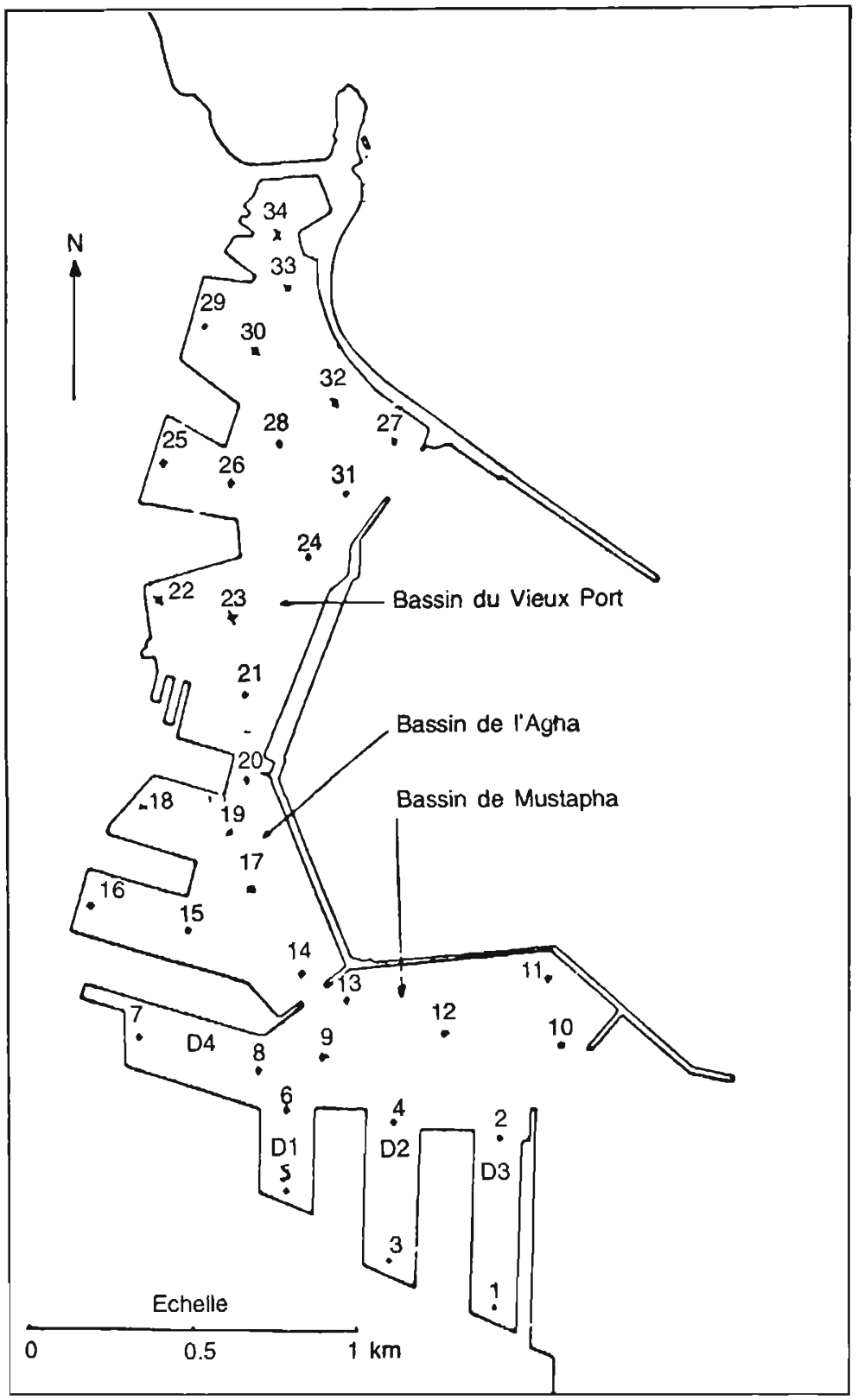

Fig. 2. - Localisation des stations. 
bre d'individus de chacune d'elle exprimé en valeur absolue (abondance) ou en valeur relative (dominance) ainsi que la densité; ceci pour estimer la richesse qualitative et quantitative du peuplement à chaque station et l'évolution, tant spatiale que temporelle, de cette richesse.

Dans le but de connaître les conditions du milieu auxquelles est soumise la macrofaune benthique installée dans chaque bassin, les paramètres physiques et chimiques (température, salinité, oxygène dissous, $\mathrm{pH}$ et matières en suspension) ont été également mesurés pour chaque station et à chaque saison.

\section{RÉSULTATS}

\section{Les peuplements des substrats meubles par bassins}

L'étude dynamique des peuplements macrobenthiques de chaque station et à chaque saison et l'étude des conditions du milieu, ont permis de mettre en évidence la spécificité de chacun des bassin.

\section{Bassin de Mustapha}

Les fonds et les sorties de darses sont azoïques toute l'année où sévit une pollution de diverses origines (chimique, organique, thermique, etc.); de plus, les apports des eaux de la baie par la passe Sud, sont sous l'influence des égouts de la partie Est de la ville et du flux de l'Oued El Harrach lui même très pollué. Ce bassin se caractérise par une température et une teneur en matière en suspension élevées et des valeurs basses de la salinité et de l'oxygène dissous.

Le peuplement est limité au niveau de l'aire d'évolution des navires; il est très peu diversifié et est constitué de deux espèces de Polychètes indicatrices de pollution 1 (IP1): Capitella capitata et Scolelepis fuliginosa. Les plus fortes densités pour l'ensemble du port ont été observées dans ce bassin dont les maximums peuvent atteindre plus de $25000 \mathrm{ind} / \mathrm{m}^{2}$ avec Capitella capitata comme principale espèce, responsable des fortes poussées quantitatives.

\section{Bassin de l'Agha}

Le peuplement de ce bassin intermédiaire est plus diversifié (maximum 20 espèces) et dense (moyenne de $4000 \mathrm{ind} / \mathrm{m}^{2}$ ) sans pour autant atteindre les pics quantitatifs du bassin de Mustapha. Le groupe faunistique dominant est celui des Polychètes et plus particulièrement des indicatrices de pollution; l'évolution saisonnière a montré une alternance de forte dominance des IP1 avec les indicatrices de pollution 2 (IP2) telles que Polydora antennata, Staurocephalus rudolphii, Nereïs caudata, Audouinia tentaculata... Cette alternance des espèces montre la compétition entre les 2 groupes IP1 et IP2 pour occuper le milieu où périodiquement l'un des groupes se substitue à l'autre. La présence des espèces à large répartition écologique (Lre) est représentée par le mollusque Corbula gibba. 
La situation intermédiaire de ce bassin révèle un état d'équilibre instable du peuplement qui peut basculer selon l'augmentation ou la diminution de la charge polluante.

\section{Bassin du Vieux Port}

Son peuplement se répartit ainsi:

- Une dominance des Lre, particulièrement Corbula gibba, indicatrice de matière organique, dans les stations des fonds de darses et dans le secteur situé à proximité du bassin de l'Agha.

- Une codominance des Lre et des espèces indicatrices d'instabilité avec respectivement Corbula gibba et Tharyx marioni en tant que principales espèces localisées aux sorties de darses et dans une partie du bassin d'évolution des navires.

- Une dominance des indicatrices d'instabilité représentées essentiellement par Tharyx marioni qui se localise dans les régions sous l'influence des eaux du large de la baie d'Alger.

Cette répartition se fait graduellement, des stations situées dans le fond des darses vers le bassin d'évolution et du bassin de l'Agha vers le passe nord.

Le peuplement se caractérise par sa richesse qualitative (33 espèces) et une densité moyenne de 2734 ind $/ \mathrm{m}^{2}$.

Les groupes écologiques les mieux représentés sont les Lre (Corbula gibba, Eunice vitata, Lumbrineris latreilli, Notomastus latericeus...); lorsque la dominance de ce groupe diminue, le groupe des indicatrices d'instabilité (avec les espèces Tharyx marioni, Abbra alba, Prionospio malmgreni et Glycera convoluta), se met en place. Une compétition interspécifique ou intergroupe est notée.

A partir de cette étude dynamique des stations, les indices de diversité (Shannon et Equitabilité) ont été déterminés; en se basant sur les travaux de Daget (1979) et Glemarec et Hily (1981), des seuils d'équilibre pour les peuplements ont été fixés ainsi: (fig. 3)

$E>0.80$ peuplement en équilibre $0.80 \geq E>0.65$ peuplement en léger déséquilibre $0.65 \geq E>0.50$ peuplement en déséquilibre $0.50 \geq E>0.00$ peuplement en déséquilibre fort $E=0.00$ peuplement inexistant

\section{Détermination des différentes zones (Figures 4 et 5 )}

En se basant sur les travaux de Reish (1959), Bellan (1967, 1984), Romano (1973), et à partir des résultats obtenus, une zonation ( 7 zones) des peuplements macrobenthiques a été définie et délimitée pour le port d'Alger. Cette zonation a été faite à partir de la composition faunistique de chaque station, de la dominance et de la signification écologique de chaque espèce ou groupe d'espèces. Elle s'inspire donc aussi des travaux de Pearson et Rosenberg (1978), Glemarec et Hily (1981).

\section{- Zone de pollution maximale (P.M.):}

C'est une zone azoïque comparable à celle définie par les auteurs cités 


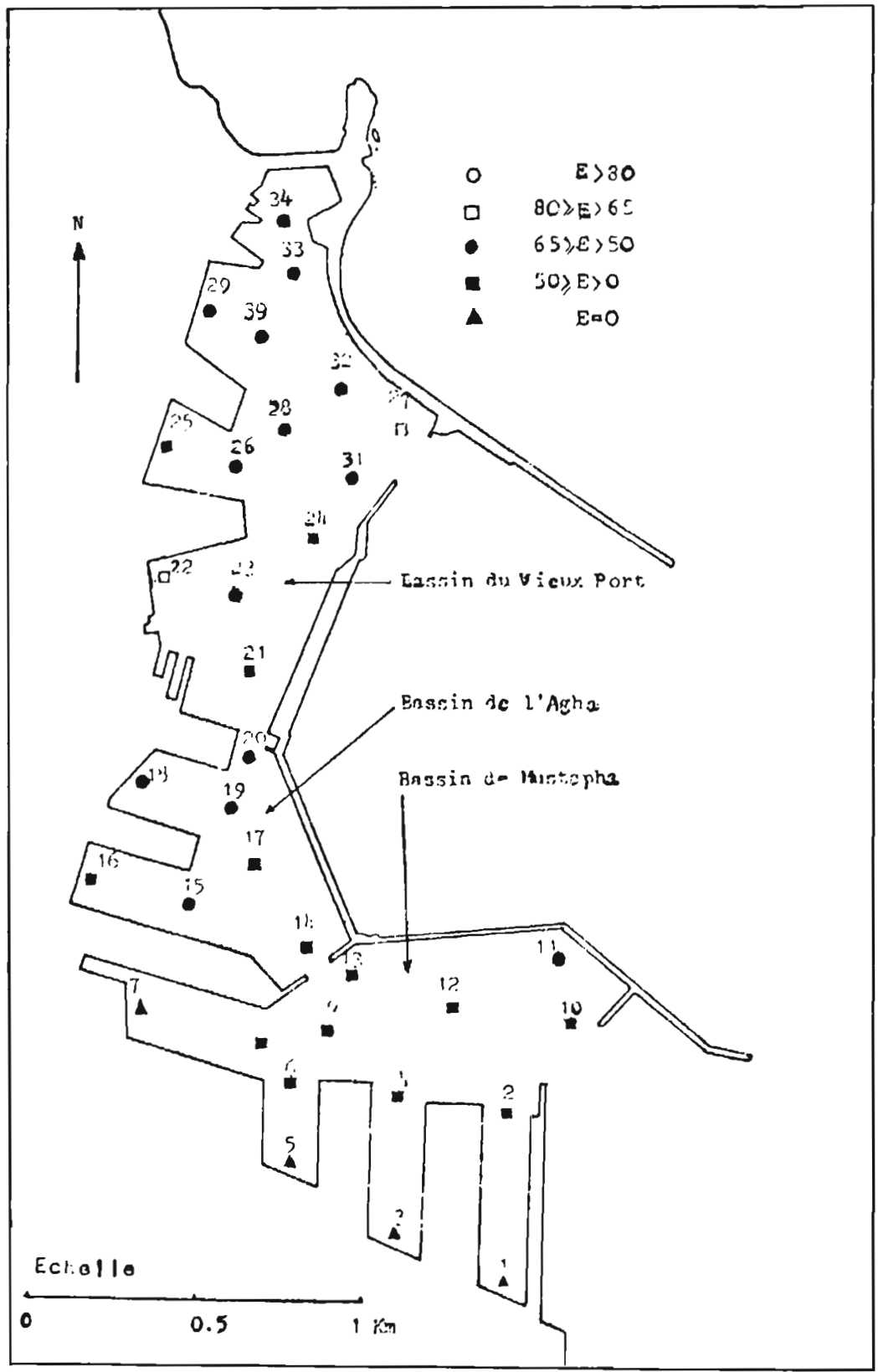

Fig. 3. - États d'équilibre du port d'Alger. 


\begin{tabular}{|c|c|c|c|c|c|c|c|}
\hline Zone & $P M$ & TI & $P$ & $T 2$ & $D$ & T3 & $N$ \\
\hline Gr. Ecol. & & I.P.I & I.P. $\mid$ & I.P. 2 & L.r.e & Ind Inst. & $N$. \\
\hline Espices & & Copitello c. & $\begin{array}{l}\text { Copitello a. } \\
\text { Solelepis } \mathrm{r} \text {. }\end{array}$ & 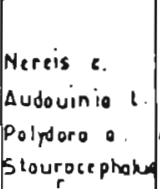 & $\begin{array}{l}\text { Corbula g. } \\
\text { Lumbrinereis } 1 \\
\text { Apsoudes } 0.0 . \\
\text { Nolomostus } 1 .\end{array}$ & $\begin{array}{l}\text { Tharyz m. } \\
\text { Abro o. } \\
\text { Glycero c. } \\
\text { Aronospio m. } \\
\text { Meleromastus }\end{array}$ & \\
\hline $\begin{array}{l}\text { Densile } \\
\text { Nor. } \\
\text { 'espèces }\end{array}$ & 1 & $100 \mathrm{ind} / \mathrm{m} / 2$ & $\begin{array}{l}10.000 \\
\text { ind. } / \mathrm{m}^{2}\end{array}$ & {$\left[\begin{array}{rr}4.000 & \\
\text { ind. } / \mathrm{m}^{2}\end{array}\right.$} & $\begin{array}{l}\text { mox. } \\
500 Q \\
\text { ind./m } 40\end{array}$ & $\mid \begin{array}{l}10000^{\circ} \\
3000 \\
\text { ind. } / \mathrm{m}^{2}\end{array}$ & $<3000$ \\
\hline 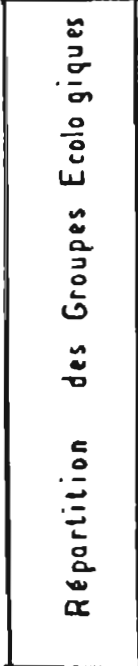 & $\begin{array}{l}-20 \% \\
-60 \% \\
-40 \% \\
-20 \%\end{array}$ & & a & and & 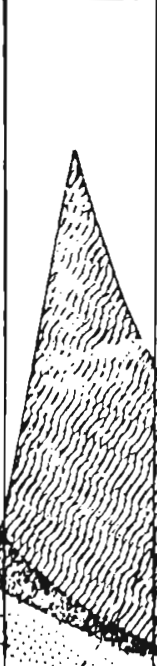 & (1) & 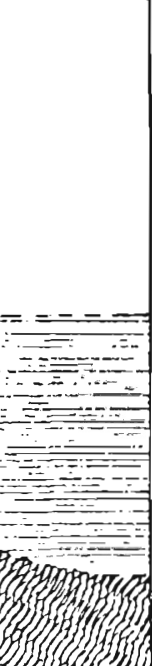 \\
\hline
\end{tabular}

Fig. 4. - Représentation graphique des zones de dégradation des peuplements macrobenthiques.

ci-dessus; le sédiment est une vase noire gluante et fétide à débris variés. - Zone de transition 1 (T1):
A la limite de la zone azoïque, elle se définit par un peuplement très peu diversifié (1 espèce) et une faible den- 


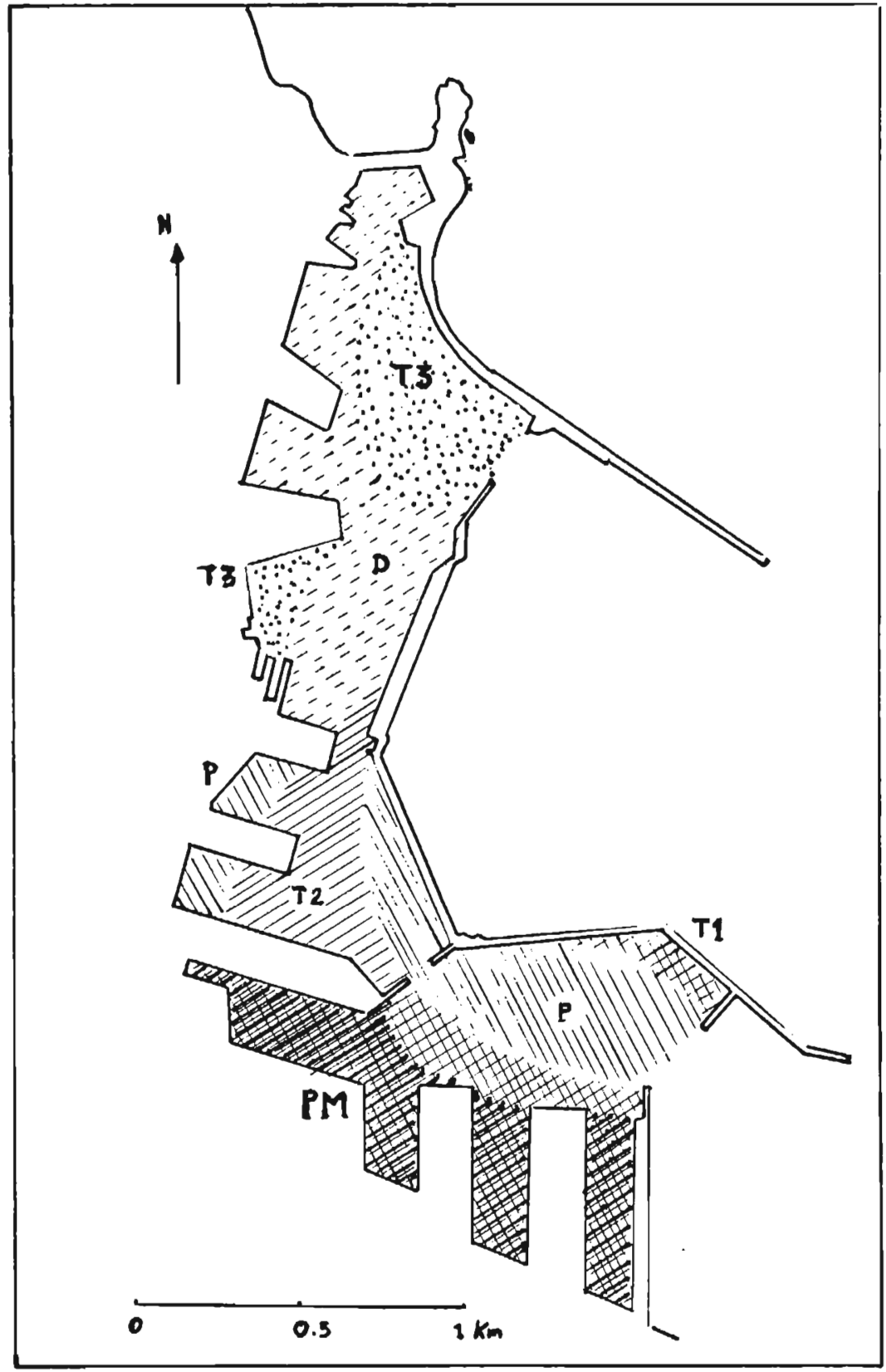

Fig. 5. - Zonation du port d'Alger. 
sité (inférieure à $100 \mathrm{ind} / \mathrm{m}^{2}$ ) et constitué par les indicatrices de pollution 1 (Capitella capitata).

\section{- Zone polluée, $(P)$ :}

Elle se caractérise par une densité qui peut atteindre des valeurs supérieures à $10000 \mathrm{ind} / \mathrm{m}^{2}$ des indicatrices de pollution 1. Capitella capitata est capable de supporter les conditions anoxiques du milieu; elle est responsable des fortes poussées quantitatives saisonnières et prolifère considérablement; elle est suivie de Scolelepis fuliginosa plus abondante lorsque les conditions du milieu ne sont pas extrêmement défavorables.

Ces espèces et plus particulièrement Capitella capitata sont considérées comme des indicatrices biologiques de pollution des conditions extrêmes du milieu. Elles ont été localisées dans le bassin d'évolution de Mustapha.

\section{- Zone de transition 2 (T2):}

Intermédiaire entre la zone polluée et la zone suivante, la zone T2 se caractérise par un peuplement moins dense (entre 1000 et $4000 \mathrm{ind} / \mathrm{m}^{2}$ ) et plus diversifié (entre 6 et 15 espèces) qui est dominé par les indicatrices de pollution. Capitella capitata et Scolelepis fuliginosa ne sont plus aussi abondantes que dans la zone polluée; elles sont concurrencées et remplacées par Nereïs caudata, PoIydora antennata, Staurocephalus rudolphi, Audouina tentaculata etc.

Ces espèces constituent le groupe des indicatrices de pollution 2 (IP2) et, lorsqu'elles s'installent, généralement de façon permanente, et à dominance supérieure à $75 \%$, elles indiquent une pollution modérée qui a été observée dans le bassin de l'Agha.

- Zone de déséquilibre (D):

Le peuplement y est dense (valeur maximale $5000 \mathrm{ind} / \mathrm{m}^{2}$ ) et beaucoup plus diversifié (nombre d'espèces maximal: 63). Le groupe écologique dominant est représenté par les espèces à large répartition écologique (Lre) et comme principale espece Corbula gibba qui représente plus de la moitié des effectifs aux stations des fonds de darses du bassin du Vieux Port.

Elle est accompagnée par Eunice vittata, E. harassi, Lumbrineris latreilli, Notomastus latericeus, Phtisica marina, Apseudes africanus orientalis et A. latreilli mediterraneus.

Dans cette zone, le niveau de pollution est moins important que dans les zones précédentes et la dominance des indicatrices de pollution est inférieure à $15 \%$.

D'autres groupes d'espèces ou groupes écologiques essaient de s'installer:

- Les Vasicoles tolérantes Tharyx marioni, Abra alba, Heteromastus filiformis et Chaetozone setosa sont constantes et abondantes; elles constituent un stock écologique non négligeable pour cette zone.

- Les espèces caractéristiques de biocenose (SFBC et SVMC) telles que les plus importantes Prionospio malmgreni, Glycera convoluta, Tellina pulchella et Phyllodoce laminosa.

Ces espèces (Vasicoles et Sabulicoles) ont été rassemblées dans le 
groupe des espèces indicatrices d'instabilité défini par Picard (1965), Bourcier et al (1984) et Nodot et al (1984).

\section{- Zone de transition 3 (T3):}

Elle a plus ou moins les mêmes caractéristiques faunistiques que la zone de déséquilibre, avec cependant deux aspects différents dans la constitution du peuplement. Une codominance ou dominance entre les Lre (Corbula gibba comme principale espèce) et les espèces indicatrices d'instabilité sont observées (principalement Tharyx marioni).

Cette zone montre que ces espèces tendent à se développer dans ce milieu et une compétition interspécifique ou intergroupe s'installe. Cette compétition, mettant en évidence l'instabilité du peuplement, peut avoir plusieurs origines:

- Les conditions du milieu,

- Le régime trophique des différentes espèces,

- La nature du substrat.

Cette compétition interspécifique ou intergroupe, signalée également par Glemarec et Hily (1981), intervient pour rééquilibrer un peuplement afin qu'il se rapproche d'un peuplement de milieu naturel.

\section{- Zone normale $(\mathrm{N})$ :}

Cette zone repose sur la connaissance d'un peuplement en conditions saines et normales.

\section{CONCLUSION}

L'ensemble des résultats ont montré la spécificité de chacun des bassins et l'état du port durant les saisons étudiées. Deux gradients de pollution décroissants ont été mis en évidence, le premier affecte tous les fonds de darses en s'étalant graduellement vers les bassins d'évolution, le second part du bassin de Mustapha, le plus pollué, traverse en décroissant le bassin de l'Agha, pour atteindre le bassin du Vieux Port le moins pollué (fig.6).

Cette modeste contribution permet de situer le problème de la pollution du port d'Alger dans sa globalité; elle doit être complétée par d'autres études qui permettront par exemple de quantifier la pollution.

Des études similaires pour plusieurs ports importants du bassin méditerranéen permettront de fixer une réglementation juridique pour la protection de l'environnement marin.

\section{REMERCIEMENTS}

Ce travail n'aurait pu être réalisé sans l'aide, aussi bien en mer qu'en laboratoire de Messieurs A. Bakalem, G. Bellan et J.-C. Romano. Pour l'aide et les conseils qu'ils m'ont prodigué, qu'ils trouvent ici, l'expression de ma profonde gratitude. 


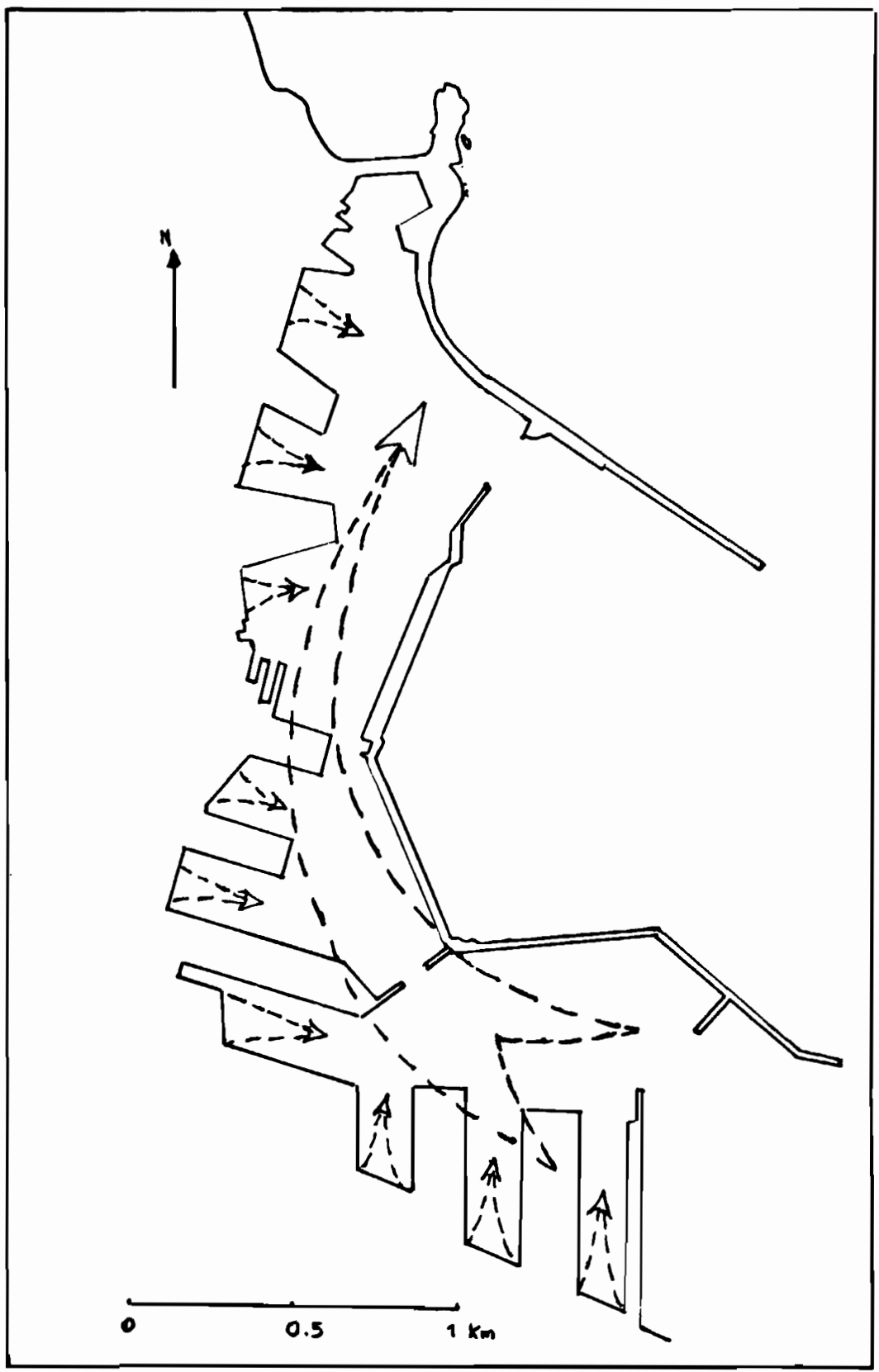

Fig. 6. - Gradients de pollution. 


\section{BIBLIOGRAPHIE}

Bakalem A. et Romano J.-C., 1983. Pollution et peuplements benthiques dans la région algéroise, (baie d'Alger, baie de Bou Ismail). VIe journ. Étud. Pollut. C.I.E.S.M., 687-697.

Bakalem A., Rebzani-Zahaf C., Romano J.-C., Tahar M.L., 1986. Cartographie des peuplements benthiques du port d'Alger. Rapport Communication int. Mer Méditerranée, 30(2), 135.

Bellan G., 1967. Pollution et peuplements benthiques sur substrats meubles dans la région de Marseille. Deuxième partie: l'ensemble portuaire marseillais. Revue int. Océanogr. med., 8, 5195.

Bellan G., 1984. Indicateurs et indices biologiques dans le domaine marin. Bull. Ecol. 15 (1), 13-20.

Bourcier M., Nodot C., Jeudy de Grissac A. et Tine J., 1984. Répartition des biocénoses benthiques en fonction des substrats sédimentaires de la rade de Toulon (France). Tethys, 11(2), 141153.

Daget J., 1979. Les modèles mathématiques en écologie Masson ed., Paris, $170 \mathrm{p}$.

Glemarec M., Hily C., 1981. Perturbations apportées à la macrofaune benthique de la baie de Concarneau par les effluents urbains et portuaires. Acta CEcol. Applic., 2(2), 139-150.

Hily C., 1984. Variabilité de la macrofaune benthique dans les milieux hypertrophiques de la rade de Brest. Thèse Doct. ès-Sc., Univ. Bretagne Occident., Brest, 359 p. + 377 p.

Nodot C., Bourcier M., Jeudy de Grissac A., Hensner S., Regis J. et Tine J. 1984. Répartition des biocénoses benthiques en fonction des résultats sédimentaires de la rade de Toulon (France). 2. La grande rade. Tethys, 11 (2): 141-153.

Picard J. 1965. Recherches qualitatives sur les biocénoses marines de substrats meubles dragables de la région marseillaise. Rec. Trav. St. mar. Endoume, 52 (36), 3, 160. 